\title{
Desenvolvimento de aplicativo móvel para o acompanhamento pré-natal e validação de conteúdo
}

Development of a mobile application for prenatal care and content validation

Desarrollo de aplicación móvil para el seguimiento de la atención prenatal y validación de contenido

Francisca Marta de Lima Costa Souza ${ }^{1}$ id https://orcid.org/0000-0002-2442-9499

Wenysson Noleto dos Santos ${ }^{1}$ id htps://orcid.org/0000-0002-2093-5415

Janmilli da Costa Dantas ${ }^{1}$ iD https://orcid.org/0000-0001-5429-6108

Helena Rangel Alves de Sousa ${ }^{1}$ (D https://orcid.org/0000-0001-7323-9782)

Olga Alice Alencar Moreira ${ }^{1}$ id https://orcid.org/0000-0002-5625-7011

Richardson Augusto Rosendo da Silva ${ }^{1}$ id https://orcid.org/0000-0001-6290-9365

Como citar:

OA, Silv RA. Des, Dans JC, Sousa HR, Moreira para o acompanhamento pré-natal e validação de

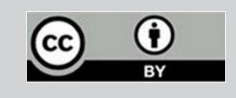

Tecnologia da informação; Gravidez; Cuidado pré-natal; Informática em enfermagem; Aplicativos móveis

Keywords

Information technology; Pregnancy; Prenatal care; Nursing informatics; Mobile application

Descriptores

Tecnología de la información; Embarazo; Atención prenatal; Informática aplicada a la enfermería; Aplicaciones móviles

Submetido

21 de Julho de 2019

Aceito

25 de Março de 2021

Autor correspondente Richardson Augusto Rosendo da Silva

E-mail: rirosendo@hotmail.com

Editor Associado (Avaliação pelos pares): Rosely Erlach Goldman (https://orcid.org/0000-0003-4011-1875) Escola Paulista de Enfermagem, Universidade Federal de São Paulo, São Paulo, SP, Brasil

\section{Resumo}

Objetivo: Desenvolver aplicativo móvel para gestantes em acompanhamento pré-natal e validar o conteúdo.

Métodos: Estudo metodológico, tecnológico de abordagem quantitativa realizado em três etapas: revisão integrativa de literatura, validação de conteúdo e construção do aplicativo. A revisão foi realizada em Bases de Dados Bibliográficas; em seguida, um instrumento guia foi elaborado abarcando assuntos sobre pré-natal, parto e puerpério, o qual passou por uma validação inicial, por meio de um grupo focal. Para a construção do aplicativo seguiram-se as fases: análise, design, desenvolvimento, implementação e avaliação de especialistas. A construção do aplicativo e validação do conteúdo foi realizada por 21 especialistas, sendo 14 enfermeiros obstetras e 7 profissionais da área de tecnologia da informação e comunicação. Utilizou-se o Índice de Validade de Conteúdo (IVC), considerando como taxa de concordância valores acima de 80\%, Concordância interavaliadores e teste não paramétrico de Mann-Whitney para verificação da fase Delphi 1 e 2 .

Resultados: 0 aplicativo possui 111 telas sobre o pré-natal, parto, puerpério e aleitamento materno, caderneta virtual da gestante, despertador como lembrete de consultas e o menu fale conosco. Após duas rodadas da técnica Delphi, obteve-se concordância entre os especialistas, com índice de validade de conteúdo médio de 0,89 .

Conclusão: 0 aplicativo obteve IVC geral adequado entre os especialistas, evidenciando que as informações abordadas e a parte técnica do sistema são confiáveis, sendo validado quanto ao conteúdo. Este apresenta-se como uma potencial ferramenta para promoção da saúde, no que concerne ao cuidado no período gravídicopuerperal.

\section{Abstract}

Objective: To develop a mobile application for pregnant women in prenatal care and validate its content.

Methods: This is a methodological and technological study with a quantitative approach, carried out in three stages: integrative literature review, content validation, and application construction. The review was carried out in bibliographic databases; then, a guide instrument was elaborated covering subjects about prenatal care, childbirth and the puerperium, which underwent an initial validation through a focus group. For the application construction, the following phases were followed: analysis, design, development, implementation and expert assessment. The application construction and content validation were carried out by 21 experts, being 14 obstetric nurses and 7 Information and Communication Technology professionals. Content Validity Index (CVI) was used, considering as an agreement rate values above $80 \%$, inter-rater agreement and Mann-Whitney non-parametric test to verify Delphi 1 and 2 phases. 
Results: The application has 111 screens about prenatal care, childbirth, puerperium and breastfeeding, the pregnant woman's virtual notebook, an alarm clock as a reminder of appointments and the contact us menu. After two rounds Delphi technique rounds, agreement was reached between the experts, with an average Content Validity Index of 0.89 .

Conclusion: The application obtained an adequate general CVI among experts, showing that the information covered and the system's technical part are reliable, being validated in terms of content. This presents itself as a potential instrument for health promotion with regard to care in the pregnancy-puerperal period.

\section{Resumen}

Objetivo: Desarrollar aplicación móvil para gestantes para el seguimiento de la atención prenatal y validar el contenido.

Métodos: Estudio metodológico, tecnológico de enfoque cuantitativo realizado en tres etapas: revisión integradora de literatura, validación de contenido y elaboración de la aplicación. La revisión fue realizada en bases de datos bibliográficas. Luego, se elaboró un instrumento guía sobre temas relacionados con control prenatal, parto y puerperio, que pasó por una validación inicial, por medio de un grupo focal. Para elaborar la aplicación, se siguieron las siguientes fases: análisis, diseño, desarrollo, implementación y evaluación de especialistas. La elaboración de la aplicación y la validación del contenido fueron realizadas por 21 especialistas, de los cuales 14 eran enfermeros obstetras y siete profesionales del área de tecnologías de la información y la comunicación. Se utilizó el Índice de Validez de Contenido (IVC), considerando como índice de concordancia valores superiores a 80 \%, concordancia interevaluadores y prueba no paramétrica de Mann-Whitney para verificación de la fase Delphi 1 y 2.

Resultados: La aplicación posee 111 pantallas sobre control prenatal, parto, puerperio y lactancia materna, libreta virtual de gestante, alarma como recordatorio de consultas y el menú "contáctenos". Luego de dos rondas del método Delphi, se obtuvo concordancia entre los especialistas, con un índice de validez de contenido promedio de 0,89 .

Conclusión: La aplicación obtuvo un IVC general adecuado entre los especialistas, lo que deja en evidencia que la información abordada y la parte técnica del sistema son confiables y, de esta forma, el contenido es validado. Se presenta como una herramienta potencial para la promoción de la salud, en lo que respecta al cuidado durante el embarazo y el puerperio.

\section{Introdução}

As Tecnologias da Informação e Comunicação (TICs) são um conjunto de recursos tecnológicos e computacionais dedicados ao armazenamento, processamento e comunicação da informação, apresentando-se como um forte aliado ao setor da saúde, principalmente quando se almeja a promoção à saúde da população. $\mathrm{O}$ seu potencial, quando aplicado ao cuidado em saúde de pacientes, tem importância crítica e estratégica na medida em que amplia a concepção de ambiente do cuidado - um espaço móvel de interaçóes interligando contextos, sujeitos e saberes, em que cuidar e educar, caminhando juntos, ganham destaque e relevância. ${ }^{(1,2)}$

As TICs já fazem parte da vida cotidiana de muitos pacientes e têm o potencial de impactar a promoção à saúde de forma positiva, por meio de orientaçóes de prevenção e tratamento de doenças e agravos, bem como na manutenção de hábitos de vida saudáveis. Outrossim, podem promover maior aproximação com o profissional e melhorar a compreensão do usuário sobre seu estado de saúde, além de aguçar o seu interesse em cuidar da própria qualidade de vida. ${ }^{(3)}$

As tecnologias móveis em saúde, mobile health, são definidas como medicina ou saúde pública pra- ticada por meio de dispositivos móveis, como telefones celulares, aparelhos de monitoramento de pacientes, assistentes pessoais digitais e outros dispositivos sem fio. ${ }^{(2)}$ A saúde móvel foi reconhecida pela Organização Mundial da Saúde (OMS), em 2011, como uma potencial estratégia para as práticas de saúde, favorecendo a incorporação desse artefato de forma cada vez mais freqüente. ${ }^{(3)}$

Assim, destacam-se as tecnologias móveis, como celulares, tablets, smartphones, dentre outros, e com eles a utilização de Aplicativos Móveis (App). Todas essas alternativas constituídas pelas tecnologias são importantes para promover a saúde em diversas áreas ou programas de saúde, por exemplo, o pré-natal.

A assistência pré-natal tem como principal objetivo assegurar o bom desenvolvimento da gestação, por meio dos cuidados, educação e aconselhamento, contribuindo para o parto e nascimento saudáveis. No entanto, a existência de falhas na assistência pré-natal, tais como barreiras no acesso, início tardio e realização incompleta dos procedimentos preconizados, dificulta a identificação e tratamento das principais intercorrências no ciclo gravídico hipertensão arterial, hemorragias e infecções -, afetando a qualidade e efetividade do pré-natal. ${ }^{(4,5)}$

Ressaltam-se, ainda, diversos obstáculos para a não realização do pré-natal evidenciando as desi- 
gualdades sociais que persistem no país, com menor acesso das mulheres indígenas e negras, menor escolaridade e residentes nas regióes Norte e Nordeste. A falta de vínculo e articulação entre os serviços que prestam assistência pré-natal e ao parto é outro problema identificado, resultando na peregrinação da gestante na rede assistencial. ${ }^{(5)}$

Tais problemas favorecem os elevados índices de mortalidade materna, complicações no momento do parto e nascimento de crianças prematuras, decorrentes da falta de diagnóstico e tratamento de complicações gestacionais. ${ }^{(6)}$ Nesse contexto, o enfermeiro, enquanto profissional de saúde, pode colaborar na assistência pré-natal desenvolvendo açóes de promoção à saúde e prevenção de complicaçóes no período gravídico-puerperal por meio da utilização de tecnologias inovadoras em saúde. ${ }^{(4)}$

Os aplicativos móveis disponíveis para gestantes, encontrados até o presente momento, são direcionados apenas ao controle da hipertensão, diabetes e peso; prevenção de parto prematuro e infecção do trato urinário; e redução do tabagismo e etilismo. ${ }^{(6-12)}$

Dessa forma, observou-se a necessidade de construir um App contendo informações sobre o pré-natal, parto, puerpério e aleitamento materno, bem como disponibilizar a caderneta virtual da gestante, alarme para lembrete de consultas de acompanhamento pré-natal e um menu fale conosco para esclarecimento de dúvidas. Ante o exposto, o objetivo deste estudo foi desenvolver aplicativo móvel para gestantes em acompanhamento pré-natal e validar o conteúdo.

\section{Métodos}

O estudo foi aprovado pelo Comitê de Ética em Pesquisa da Universidade Federal do Rio Grande do Norte (UFRN) sob parecer no 2.356.769. Trata-se de um estudo metodológico, tecnológico de abordagem quantitativa, realizado no período de janeiro a dezembro de 2018. Este foi composto por três etapas: 1) revisão integrativa de literatura, 2) construção do aplicativo e 3) validaçáo do conteúdo do aplicativo por enfermeiros obstetras e a usabilidade e aplicabilidade da ferramenta tecnológica por profissionais da área da Tecnologia da Informação e Comunicação, os quais atuaram como especialistas.

Inicialmente, realizou-se uma revisão integrativa de literatura para selecionar o conteúdo a ser disponibilizado no aplicativo, partindo-se da seguinte questão norteadora: quais recomendaçóes devem ser fornecidas às mulheres durante o pré-natal sobre gravidez, parto e puerpério? Assim, o conteúdo foi buscado nas bases de dados Literatura LatinoAmericana e do Caribe em Ciências da Saúde, Bases de Dados em Enfermagem, Scientific Eletronic Library Online, Science Direct, Cummulative Index to Nursing and Allied Health Literature, Cochrane, Web of Science, Scopus e Medical Literature Analysis and Retrievel System Online (Medline). Utilizaramse os Descritores em Ciências da Saúde (DeCS) e Medical Subject Heading (MeSH), respectivamente: "Cuidado Pré-Natal", "Gravidez", "Saúde da Mulher", "Enfermagem", "Parto", "Período PósParto”, "Prenatal Care”, "Pregnancy”, “Women's Health" e "Nursing”, "Parturition", "Postpartum Period". Estes foram cruzados por meio do operador booleano AND, a partir dos seguintes cruzamentos 3 X 3: "Cuidado Pré-Natal” AND "Gravidez" AND "Saúde da Mulher"; "Cuidado Pré-Natal" AND "Gravidez" AND "Enfermagem"; "Gravidez" AND "Saúde da Mulher" AND "Enfermagem"; "Gravidez" AND "Parto" AND "Período PósParto"; "Gravidez" AND "Parto" AND "Cuidado Pré-Natal"; "Gravidez" AND "Parto" AND "Saúde da Mulher"; "Gravidez" AND "Parto" AND "Enfermagem"; "Prenatal Care" AND "Pregnancy" AND "Women's Health"; "Prenatal Care" AND "Pregnancy" AND "Nursing"; "Pregnancy" AND "Women's Health" AND "Nursing";"Pregnancy" AND "Parturition" AND "Postpartum Period"; "Pregnancy" AND "Parturition" AND "Prenatal Care"; "Pregnancy" AND "Parturition" AND "Women's Health"; "Pregnancy" AND "Parturition" AND "Nursing".

Como critério de inclusão, elencaram-se estudos que abordassem recomendaçôes sobre o pré-natal, parto e puerpério; e critério de exclusão, artigos disponíveis em idiomas diferentes do inglês, português e espanhol. Ressalta-se que de forma complementar utilizou-se a literatura cinzenta, incluindo-se docu- 
mentos governamentais e de sociedades de especialistas, tais como manuais e protocolos assistenciais.

Após a leitura dos artigos e materiais selecionados na revisão integrativa de literatura, elaborou-se um instrumento guia abarcando assuntos relacionados ao pré-natal, parto e puerpério. Esse instrumento consistiu em um roteiro com conteúdos extraídos da revisão de literatura realizada na etapa inicial. Este passou por um processo de validação inicial, quanto ao conteúdo, por meio de um grupo focal composto por oito professores universitários de uma instituição federal e estadual de ensino, os quais eram enfermeiros obstetras. $\mathrm{O}$ tipo de validação foi por consenso. Nessa fase, a validação de conteúdo do instrumento guia foi importante para selecionar de forma mais acurada os conteúdos fundamentais que deveriam compor o aplicativo Gestação Saudável. Essa etapa foi fundamentada apenas na definição dos conteúdos.

A construção do aplicativo baseou-se no modelo Design Instrucional Contextualizado (DIC), que recomenda as seguintes etapas: análise, design, desenvolvimento, implementação e avaliação de especialistas. ${ }^{(13)}$

A etapa da análise consiste em entender o problema educacional e elaborar uma solução relacionada. Esta envolveu o levantamento das necessidades, a caracterização do público-alvo, a definição dos objetivos, a coleta do referencial bibliográfico, a análise da infraestrutura tecnológica e a criação de um fluxograma. ${ }^{(13)}$ Para determinar o levantamento das necessidades, foi realizada uma pesquisa de campo no intuito de identificar as principais dúvidas e quais informaçôes eram importantes serem recebidas pelas gestantes no decorrer do período gravídico-puerperal. Assim, adotou-se a técnica do grupo focal (GF) composto por 24 gestantes que realizavam o acompanhamento pré-natal em uma Unidade de Saúde da Família em um município do Nordeste do Brasil. Ocorreram dois encontros, com duração média de uma hora, cada um composto por 12 gestantes. $\mathrm{O}$ discurso emergido foi gravado, transcrito e analisado.

Como o referencial adotado no estudo envolve a etapa de caracterização do público-alvo ${ }^{(13)}$ procedeu-se consultas ao banco de dados SisPreNatal/
DATASUS. Este acesso ocorreu em 2018, no município onde realizou-se o GF, possibilitando a identificação das seguintes variáveis das gestantes: idade, escolaridade, número de filhos, início do pré-natal, número de consultas realizadas, exames realizados, vacinação e consulta puerperal.

Em seguida traçou-se o objetivo de construir um aplicativo móvel e procedeu-se com a coleta do referencial bibliográfico por meio da revisão integrativa, já descrita anteriormente. $\mathrm{Na}$ análise da infraestrutura tecnológica foi definida a estrutura da interface do aplicativo, a sequência do conteúdo e seleção de mídias. Assim, o conteúdo foi transformado em material didático, compatível com o formato de aplicativo, de modo atrativo para o público-alvo, utilizando imagens. E, por fim, desenvolveu-se um fluxograma para nortear a construção da ferramenta e, após, transformar a ideia em produto (Figura 1).

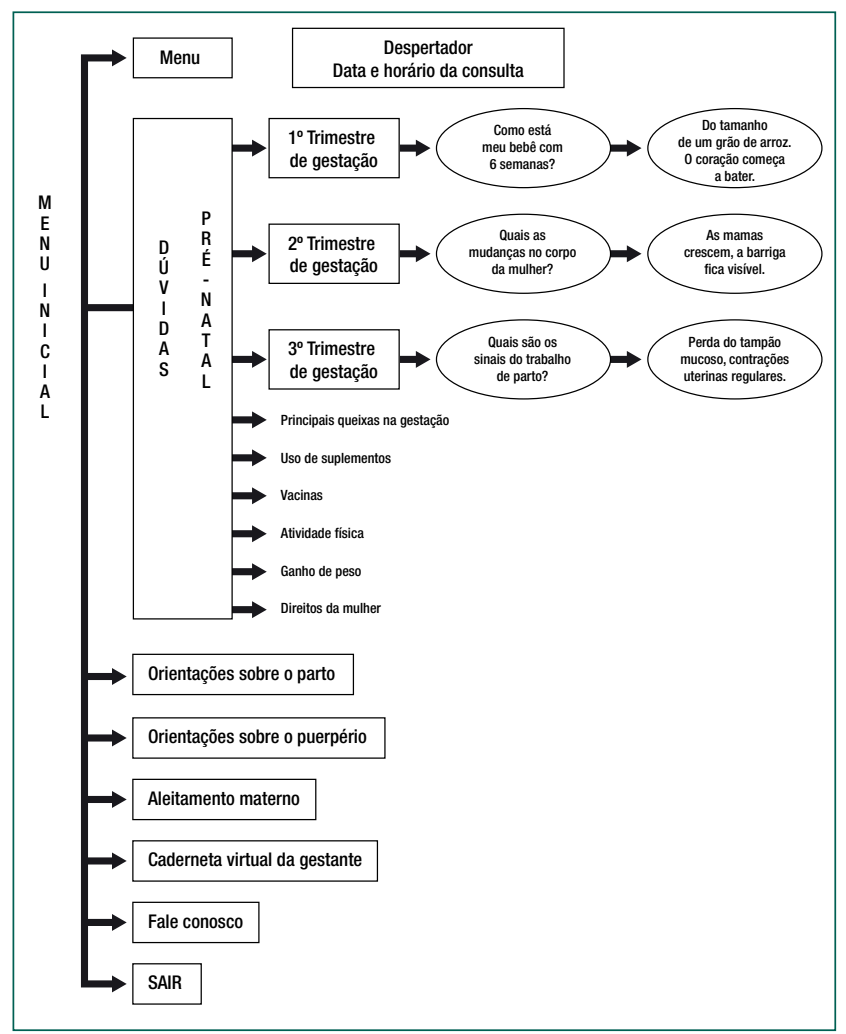

Figura 1. Representação gráfica das telas do aplicativo Gestação Saudável

A figura 1 representa o fluxograma do aplicativo "Gestação Saudável” com seus menus e ilustra a dú- 
vida mais frequente em cada trimestre da gestação, identificada em pesquisa de campo realizada na etapa de levantamento das necessidades, com o mesmo grupo focal descrito anteriormente.

O design utilizado na construção do aplicativo envolveu o planejamento e a produção do conteúdo didático, a seleção das mídias e o desenho da interface (layout). ${ }^{(13)} \mathrm{O}$ desenvolvimento compreendeu a seleção das ferramentas do aplicativo, a definição da estrutura de navegação e o planejamento da configuração de ambientes. A implementação incluiu o uso de recursos tecnológicos, a configuração das ferramentas e a instalação no dispositivo móvel. A avaliação apresentou a análise de especialistas em relação aos conteúdos, recursos didáticos e interface do ambiente. ${ }^{(13)}$

O protótipo foi elaborado por um profissional da área de tecnologia, por meio da ferramenta Android Studio, utilizando-se a linguagem de programação Java, apoiado pelos seguintes frameworks: 1) Java Server Faces-JSF, um framework Java para a Web; 2) Spring, um framework para o gerenciamento dos módulos da aplicação e dependências; e 3) Hibernate, um framework para o mapeamento objeto-relacional na base de dados. ${ }^{(13)}$

Quanto à linguagem utilizada na interface, seguiram-se as diretrizes - simplicidade, clareza e objetividade -, tornando os conteúdos acessíveis às gestantes. $\mathrm{O}$ sistema desenvolvido foi nominado "Gestação Saudável". Com a construção do aplicativo finalizada, iniciou-se a validação da aplicabilidade e usabilidade. Para tanto, utilizou-se a técnica Delphi, com nível de consenso de $80 \% .^{(13,14)}$

Em relação à quantidade de juízes, adotou-se, no presente estudo, a sugestão do mínimo de seis especialistas para validação de tecnologias e instrumento. ${ }^{(15)}$ No entanto, ressalta-se que não existe um consenso na literatura internacional sobre o número mínimo de juízes, e sim a concordância da importância da experiência clínica na formação de um perfil de expertise, bem como a necessidade de equilíbrio entre experiência e formação acadêmica sólida. ${ }^{(16)}$

Enviaram-se e-mails para 48 enfermeiros obstetras, obtendo-se retorno de 26 na primeira rodada e 14 na segunda, e 43 convites para os profissionais de tecnologia, havendo 28 participantes na primeira rodada e 7 na segunda. A devolução dos formulários de avaliação ocorreu dentro do prazo estipulado de 30 dias. A seleção dos especialistas ocorreu por meio da plataforma Lattes e o contato via carta-convite através de correio eletrônico. Os especialistas que participaram do estudo assinaram o Termo de Consentimento Livre e Esclarecido (TCLE).

Os critérios de inclusão para os enfermeiros especialistas foram: ser especialista em enfermagem obstétrica; mestre ou doutor em enfermagem; docente de instituição de ensino superior em obstetrícia ou saúde da mulher; atuação anterior na Estratégia Saúde da Família ou maternidade; e autoria de trabalhos publicados em periódicos sobre cuidado pré-natal. Já para os profissionais da área da Tecnologia da Informação e Comunicação/especialistas, utilizaram-se os seguintes critérios: graduado em área de ciência e tecnologia; especialistas na área de tecnologia da informação e comunicação; mestre ou doutor na área de ciência e tecnologia; autoria de trabalhos publicados em periódicos sobre aplicativos móveis em saúde; e docente de instituição de ensino superior. Adotou-se como critério de exclusão, para ambos, a não atualização do currículo nos últimos seis meses.

Para a coleta de dados, elaboraram-se dois formulários distintos no serviço de armazenamento do Google Drive ${ }^{\circledR}$. Um formulário foi direcionado para o grupo de enfermeiros que realizaram a avaliação de conteúdos; e o outro formulário foi enviado para o grupo de especialistas da área da Tecnologia da Informação e Comunicação que avaliaram a usabilidade e aplicabilidade do aplicativo.

O formulário destinado ao grupo de enfermeiros abarcava os conteúdos de pré-natal, parto, puerpério e aleitamento materno que compuseram o aplicativo, sendo avaliados os seguintes aspectos: clareza da linguagem, relevância e importância prática.

Já o formulário direcionado aos especialistas da área da Tecnologia da Informação e Comunicação avaliou as dimensóes "Organização das telas", "Interface das telas", "Conteúdo do sistema" e "Técnico" referentes à funcionalidade - permite facilidade no uso e precisão na execução de suas fun- 
çôes; confiabilidade - reage adequadamente quando ocorrem falhas e informa ao usuário a entrada de dados inválidos; eficiência - o tempo de execução e os recursos disponibilizados são adequados; e usabilidade - é fácil entender o conceito e sua aplicabilidade, bem como aprender a usá-lo. ${ }^{(17)}$

Para cada conteúdo apresentado no formulário, inseriu-se a escala Likert contendo cinco pontos: 1 =totalmente inadequado; $2=$ moderadamente inadequado; 3=nível de adequação que se assemelhasse ao de inadequação; $4=$ moderadamente adequado; e $5=$ totalmente adequado. ${ }^{(18)}$

Quando os especialistas não concordavam com algum item do aplicativo era possível propor um novo enunciado ou novo título ao menu; recomendar a inclusão de alguma informação adicional; ou sugerir a inclusão de novo item. ${ }^{(18)}$ Totalizaram-se duas rodadas Delphi para todos os especialistas, que foram finalizadas após concordância unânime.

Os dados foram organizados no Microsoft Excel ${ }^{\circ}$ 2016, por meio de dupla inserção, para a garantia da fidedignidade dos mesmos. Calculou-se o Índice de Validade de Conteúdo (IVC), considerando o número de especialistas que participaram do estudo e o valor aceitável do IVC para cada item, o qual variou entre 0,80 e 1,00. Destaca-se que o escore mínimo aceitável para a validade de conteúdo foi de $0,80 .^{(14)}$

Também se calculou o Índice de Fidedignidade (reliability) ou concordância interavaliadores (IRA), considerando-se como ideais, para ambos, valores iguais ou superiores a 0.80 . Além disso, no comparativo entre as duas fases Delphi, utilizou-se o teste não paramétrico de Mann-Whitney com o intuito de verificar se os resultados da fase Delphi 2 foram mais satisfatórios do que na fase Delphi 1 (comparando o Índice de Validade de Conteúdo dos domínios e diversos aspectos do questionário). $\mathrm{O}$ nível de significância foi de $5 \% .{ }^{(19)}$

O aplicativo foi desenvolvido de forma compatível com aparelhos de sistema operacional Android na versão 1.0. A escolha ocorreu pelo fato de este, na realidade brasileira, ser o tipo de sistema compatível com a maior parte dos aparelhos celulares, de modo que mais gestantes possam ter acesso à ferramenta tecnológica. ${ }^{(20)}$

\section{Resultados}

Para o desenvolvimento do aplicativo, selecionaram-se 92 artigos para leitura na íntegra, dos quais 29 foram incluídos no estudo. Verificou-se que a maioria dos estudos foi encontrada na base de dados Medline (60\%), publicados a partir do ano 2016 (34\%), realizados no exterior, cujo idioma era o inglês (73\%).

Constatou-se que os artigos traziam as seguintes informações que poderiam compor um aplicativo móvel para promover o cuidado de mulheres na gestação: modificações fisiológicas da gravidez e desenvolvimento do feto; queixas no período gravídico e puerperal; uso de suplementos, vacinas, atividade física e ganho de peso; direitos da mulher grávida, parto, benefícios do parto humanizado e nascimento, puerpério, aleitamento materno; e dúvidas do pré-natal.

Com acesso ao aplicativo "Gestação Saudável", o usuário navegará nas interfaces para obter as informaçóes de saúde quanto ao pré-natal, parto, puerpério e aleitamento materno. As gestantes poderão fazer questionamentos durante todo o período gestacional por meio do menu "Fale Conosco". Desse modo, para garantir a comunicação, fizeram-se o registro no Google e a hospedagem do aplicativo para que ele funcione tanto offline como online.

A tela inicial do aplicativo possui uma funcionalidade de acesso a um menu com sete opçóes principais: informaçôes sobre a gravidez e cuidados no pré-natal, orientações sobre o parto, puerpério, aleitamento materno, data das consultas, caderneta virtual da gestante e o fale conosco (Figuras 2 e 3).

No primeiro link, "informaçóes sobre a gravidez e cuidados no pré-natal”, o usuário terá acesso às informaçôes sobre as modificaçôes fisiológicas da gravidez e o desenvolvimento do feto por trimestre de gestação, além de fotos que ilustram seu crescimento. Essa seção também fornece informações sobre as principais queixas no período da gravidez, uso de suplementos, vacinas, atividade física, ganho de peso e os direitos da mulher grávida.

No segundo hiperlink, "orientaçóes sobre o parto”, as gestantes conhecerão os benefícios do parto humanizado, assim como os dez itens das boas prá- 


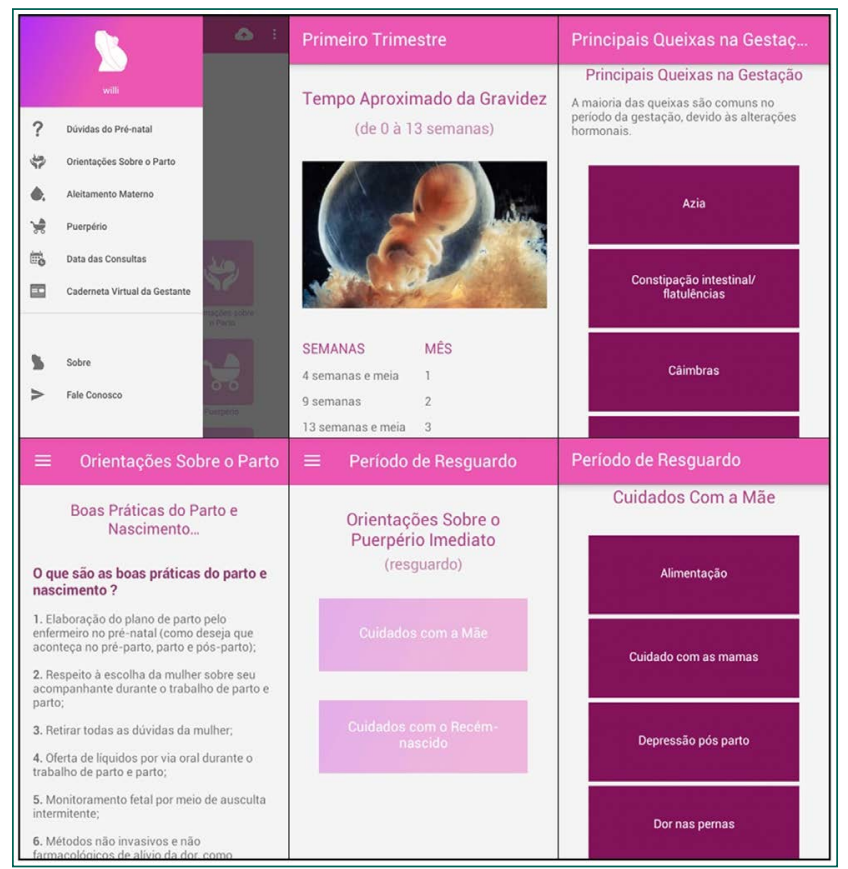

Figura 2. Representação gráfica das telas do aplicativo Gestação Saudável

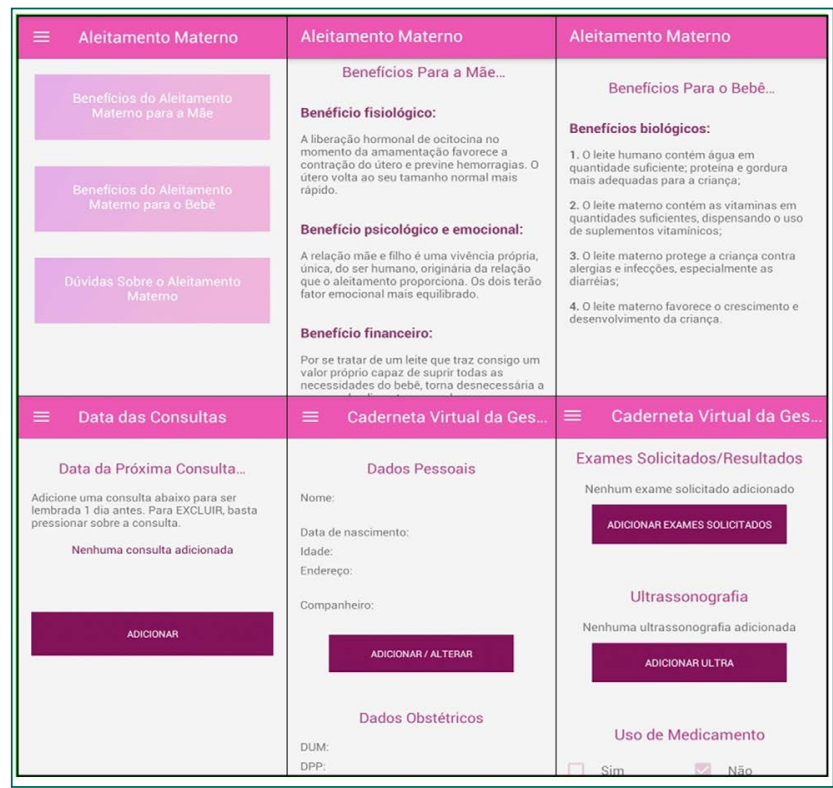

Figura 3. Representação gráfica das telas do aplicativo Gestação Saudável

ticas do parto e nascimento, com ênfase na elaboração do plano de parto.

O terceiro ícone, "puerpério", traz orientaçōes para a mulher, tais como alimentação, cuidados com as mamas, dores nos membros inferiores, higiene, ingesta de líquidos, sangramento vaginal, sinais de infecçóes, planejamento reprodutivo e de- pressão pós-parto. Já para o recém-nascido, reforça informaçóes sobre consultas de crescimento e desenvolvimento, higiene, limpeza do coto umbilical, testes de triagem neonatal e vacinas.

A quarta opção, "aleitamento materno", mostra benefícios da amamentação para a mãe, o recém-nascido e as principais dúvidas sobre o aleitamento materno.

O quinto botão, "data das consultas, despertador", servirá para registro da data ao horário da consulta de pré-natal, em que, com 24 horas antes da consulta, a gestante será lembrada por meio do despertador.

O sexto ícone, a tela "caderneta virtual da gestante", permitirá que a mulher registre suas próprias informaçóes de saúde, coletadas em cada consulta, armazene-as e rastreie-as quando for necessário.

O sétimo link, "fale conosco", tem características inovadoras, uma vez que as mulheres serão informadas sobre a possibilidade de enviar dúvidas sobre a gravidez, por meio de mensagens/e-mail, aos profissionais de saúde que farão parte da equipe de Matriciamento do Núcleo Ampliado de Saúde da Família e Atenção Básica (NASF-AB) do município de Parnamirim/RN (local da pesquisa). Para isso, por meio do link no aplicativo, as gestantes terão acesso a um e-mail institucional da secretaria de saúde, o qual os profissionais que estiverem inseridos no NASF-AB visualizarão no decorrer do seu expediente de trabalho.

O NASF-AB, nesse município, é composto pelos seguintes profissionais: enfermeiro, psicólogo, nutricionista, médico, educador físico, assistente social e terapeuta ocupacional. Eles responderão às perguntas feitas pelas gestantes via e-mail, de segunda a sexta-feira, nos seguintes horários: 08:00 às 12:00 e 14:00 às 17:00 horas. Como esses profissionais não trabalham em regime de plantáo, as questôes enviadas fora do horário de expediente serão respondidas no dia seguinte. Já as perguntas que serão realizadas aos sábados, domingos ou feriados terão suas respostas até o próximo dia útil. Quanto às participantes da pesquisa que não possuírem e-mail, estas serão orientadas e auxiliadas pelos pesquisadores a fazer um e-mail no momento da instalação do aplicativo no celular. Estratégias de assistência 
à saúde como essa podem trazer maior segurança à mulher, diminuindo, assim, o risco de complicaçóes para ela e o feto. Ressalta-se que o aplicativo pode ser instalado em qualquer celular e local, não necessitando fazer parte da pesquisa. Assim, qualquer gestante poderá enviar dúvidas e receber respostas.

Os enfermeiros especialistas que validaram o aplicativo quanto ao conteúdo eram, em sua maioria, mulheres $(70 \%)$ com idades que variaram entre 30 e 40 anos (60\%), doutores ou mestres (60\%) e que atuam apenas na docência (70\%). Estes avaliaram 16 itens referentes ao aplicativo, os quais estáo descritos na tabela 1.

Na primeira rodada Delphi, os enfermeiros especialistas sugeriram ajustes em 6 dos 16 itens avaliados, descritos na tabela 1. Aconselharam-se modificaçóes nas telas dois, cinco e sete, referentes à linguagem e conteúdo. $\mathrm{O}$ texto foi alterado de forma a evidenciar que nem todas as mulheres apresentam alteraçóes nas mamas no primeiro trimestre de gravidez e que é importante usar protetor solar e chapéu durante a exposição prolongada ao sol, de forma a evitar manchas na pele; incluir que nem todas as gestantes sentirão os movimentos do feto com 16 semanas de gestação; e adicionar, na lista da caderneta virtual da gestante, o exame de eletroforese de hemoglobina.

Os especialistas da área da Tecnologia da Informação e Comunicação, que validaram o aplicativo quanto ao conteúdo, eram, em sua maioria, homens $(89 \%)$ com idades que variaram entre 30 e 40 anos (78\%) e que possuíam doutorado ou mestrado (76\%). Apenas na dimensão "Interface das telas", no item usabilidade (Tabela 2), sugeriu-se modificação em relação à cor de uma das telas, a qual estava escura, o que dificultava a leitura do conteúdo. $\mathrm{O}$ aplicativo foi finalizado com 111 telas.

Tabela 1. Concordância dos enfermeiros obstetras acerca dos itens do aplicativo Gestação Saudável das duas rodadas Delphi

\begin{tabular}{|c|c|c|c|c|c|c|c|c|c|c|c|}
\hline \multirow{2}{*}{ Item } & \multicolumn{3}{|c|}{ IVC* do item } & \multicolumn{2}{|c|}{ IRA $^{* *}$} & \multirow{2}{*}{ Item } & \multicolumn{3}{|c|}{ IVC" do item } & \multicolumn{2}{|c|}{ IRA $^{* *}$} \\
\hline & Delphi 1 & Delphi 2 & p-valor ${ }^{* \star *}$ & Delphi 1 & Delphi 2 & & Delphi 1 & Delphi 2 & p-valor ${ }^{* * *}$ & Delphi 1 & Delphi 2 \\
\hline \multicolumn{4}{|c|}{ Tela 1- Boas-vindas e parabéns pela gestação } & \multirow{4}{*}{0,900} & \multirow{4}{*}{0,920} & \multicolumn{4}{|c|}{ Tela 9- Controle do peso na gestação } & \multirow{4}{*}{1,000} & \multirow{4}{*}{1,000} \\
\hline 11 & 0,963 & 0,998 & \multirow[t]{3}{*}{0,001} & & & 11 & 0,901 & 0,963 & \multirow[t]{3}{*}{0,012} & & \\
\hline 12 & 0,912 & 1,000 & & & & 12 & 0,844 & 1,000 & & & \\
\hline 13 & 0,941 & 1,000 & & & & 13 & 0,826 & 0,944 & & & \\
\hline \multicolumn{4}{|c|}{ Tela 2- Dúvidas do primeiro trimestre de gravidez } & \multirow{4}{*}{1,000} & \multirow{4}{*}{1,000} & \multicolumn{4}{|c|}{ Tela 10- Direitos das mulheres } & \multirow{4}{*}{0,904} & \multirow{4}{*}{0,989} \\
\hline 11 & 0,726 & 0,986 & \multirow[t]{3}{*}{0,046} & & & 11 & 0,897 & 0,984 & 0,001 & & \\
\hline 12 & 0,707 & 1,000 & & & & 12 & 0,845 & 0,973 & & & \\
\hline 13 & 0,852 & 0,978 & & & & 13 & 0,826 & 0,994 & & & \\
\hline \multicolumn{4}{|c|}{ Tela 3- Dúvidas do segundo trimestre de gravidez } & \multirow{4}{*}{0,900} & \multirow{4}{*}{0,990} & \multicolumn{4}{|c|}{ Tela 11- Orientações sobre o parto } & \multirow{4}{*}{0,948} & \\
\hline 11 & 0,852 & 0,997 & 0,035 & & & 11 & 0,897 & 1,000 & 0,012 & & 1,000 \\
\hline 12 & 0,951 & 0,993 & & & & 12 & 0,845 & 1,000 & & & \\
\hline 13 & 0,981 & 1,000 & & & & 13 & 0,826 & 1,000 & & & \\
\hline Tela 4- & terceiro tr & e de gravic & & & & Tela 1 & ticas do pa & lascimento & & & \\
\hline 11 & 0,870 & 0,907 & 0,038 & 0,890 & 0,992 & 11 & 0,818 & 1,000 & 0,001 & 0,870 & 0,980 \\
\hline 12 & 0,852 & 0,990 & & & & 12 & 0,835 & 1,000 & & & \\
\hline 13 & 0,963 & 1,000 & & & & 13 & 0,821 & 1,000 & & & \\
\hline Tela 5- & queixas na & & & & & Tela 1 & ícios do ale & nto matern & & & \\
\hline 11 & 0,753 & 0,979 & 0,001 & 1,000 & 1,000 & 11 & 0,897 & 0,984 & 0,001 & 0,924 & 1,000 \\
\hline$|2|$ & 0,870 & 0,981 & & & & 12 & 0,845 & 0,973 & & & \\
\hline 13 & 0,923 & 0,969 & & & & 13 & 0,826 & 0,994 & & & \\
\hline Tela 6- & lementos & & & & & Tela 1 & imediato & & & & \\
\hline 11 & 0,901 & 0,901 & 0,001 & 0,897 & 0,989 & 11 & 0,897 & 0,984 & 0,001 & 0,980 & 1,000 \\
\hline 12 & 0,844 & 0,963 & & & & 12 & 0,845 & 0,973 & & & \\
\hline 13 & 0,826 & 0,944 & & & & 13 & 0,826 & 0,994 & & & \\
\hline Tela 7- & gestação & & & & & Tela 1 & or com dat & brário & & & \\
\hline 11 & 0,701 & 0,969 & 0,023 & 0,902 & 0,989 & 11 & 0,897 & 0,984 & 0,001 & 1,000 & 1,000 \\
\hline 12 & 0,844 & 0,963 & & & & 12 & 0,848 & 0,973 & & & \\
\hline 13 & 0,826 & 0,944 & & & & 13 & 0,831 & 0,994 & & & \\
\hline Tela 8- & cia da ativ & ísica na ge & & & & Tela 1 & virtual da & & & & \\
\hline 11 & 0,801 & 0,963 & 0,042 & 0,900 & 1,000 & 11 & 0,719 & 0,964 & 0,001 & 1,000 & 1,000 \\
\hline 12 & 0,844 & 0,963 & & & & 12 & 0,725 & 0,973 & & & \\
\hline 13 & 0,826 & 0,994 & & & & 13 & 0,816 & 0,994 & & & \\
\hline
\end{tabular}

"IVC- Índice de Validade de Conteúdo; "IRA- Índice de Concordância Interavaliadores; "'Teste de Mann Whitney; I1- Linguagem clara, compreensível e adequada; I2- Conteúdo relevante; I3- Importância prática 
Tabela 2. Concordância dos juízes da área da Tecnologia da Informação e Comunicação acerca dos itens do aplicativo Gestação Saudável das duas rodadas Delphi

\begin{tabular}{|c|c|c|c|c|c|}
\hline \multirow{2}{*}{ Item } & \multicolumn{3}{|c|}{ IVC" do item } & \multicolumn{2}{|c|}{ IRA* } \\
\hline & Delphi 1 & Delphi 2 & $p$-value & Delphi 1 & Delphi 2 \\
\hline \multicolumn{6}{|c|}{ 1. Organização das telas } \\
\hline Funcionalidade & 0,944 & 1,000 & 0,001 & 0,923 & 1,000 \\
\hline Confiabilidade & 0,921 & 0,991 & & & \\
\hline Eficiência & 0,895 & 0,997 & & & \\
\hline Usabilidade & 0,913 & 1,000 & & & \\
\hline \multicolumn{6}{|c|}{ 2. Interface das telas } \\
\hline Funcionalidade & 0,929 & 1,000 & 0,047 & 0,987 & 1,000 \\
\hline Confiabilidade & 0,901 & 1,000 & & & \\
\hline Eficiência & 0,864 & 1,000 & & & \\
\hline Usabilidade & 0,738 & 0,991 & & & \\
\hline \multicolumn{6}{|c|}{ 3. Conteúdo do sistema } \\
\hline Funcionalidade & 0,944 & 1,000 & 0,029 & 1,000 & 1,000 \\
\hline Confiabilidade & 0,911 & 0,992 & & & \\
\hline Eficiência & 0,921 & 0,991 & & & \\
\hline Usabilidade & 0,896 & 1,000 & & & \\
\hline \multicolumn{6}{|l|}{ 4. Técnico } \\
\hline Funcionalidade & 0,825 & 0,999 & 0,019 & 1,000 & 1,000 \\
\hline Confiabilidade & 0,824 & 1,000 & & & \\
\hline Eficiência & 0,821 & 1,000 & & & \\
\hline Usabilidade & 0,945 & 1,000 & & & \\
\hline
\end{tabular}

IVC- Índice de Validade de Conteúdo; *^IRA- Índice de Concordância Interavaliadores; "'Teste de Mann Whitney

\section{Discussão}

Os aplicativos móveis ganharam força nos últimos anos na área da saúde. As lojas online disponibilizam uma imensa variedade de opçóes, que crescem a cada dia e vão desde sistemas fitness ao monitoramento e controle das mais diversas doenças. Logo, quando bem elaborados e utilizados, são ferramentas didáticas que podem trazer benefícios para a população e profissionais da saúde, ${ }^{(6)}$ acesso mais rápido dos usuários aos serviços de saúde, rapidez nos exames laboratoriais e de imagem, diagnóstico e tratamento das doenças. ${ }^{(2)}$

O aplicativo "Gestação Saudável” foi desenvolvido com o propósito de facilitar o acesso às gestantes sobre informaçóes importantes na fase do ciclo gravídico-puerperal, auxiliando-as a mitigar as dúvidas que possam surgir durante esse processo, facilitando o contato com os profissionais de saúde, a adesão às consultas de pré-natal e, consequentemente, atenuando os riscos de mobimortalidade materna por causas preveníveis.

O uso dos aplicativos aumentam a satisfação das gestantes com o atendimento pré-natal e promovem o parto normal e a maternidade segura, reduzindo, dessa forma, a morbimortalidade materna; são úteis no acompanhamento das alteraçôes - como hipertensão, sobrepeso e ansiedade materna - e na adesão ao consumo de alimentos variados e saudáveis; são ferramentas adequadas para incentivar as práticas do autocuidado das gestantes. ${ }^{(21-25)} \mathrm{O}$ fortalecimento do autocuidado pelas gestantes foi enfatizado em vários itens no aplicativo "Gestação Saudável", destacando-se no uso de suplementos, na prática da atividade física e no controle do peso.

No acompanhamento pré-natal, o uso de aplicativos, possibilitou às mulheres o enfrentamento de barreiras de acesso aos cuidados, promoveu o aumento da frequência de consultas e ajudou na identificação dos riscos à saúde das gestantes, colaborando com uma gestação, parto e nascimento saudáveis. ${ }^{(5-7)}$

O uso de aplicativos móveis também tem sido direcionado ao grupo de mulheres em pré-natal de alto risco, como descrito na pesquisa quantitativa desenvolvida nos Estados Unidos, utilizando o método paradigma da pesquisa de decisão comportamental. Recrutaram-se 16 gestantes em um ambulatório de um importante hospital acadêmico, especializado em gravidez de alto risco, a fim de testarem o aplicativo My Healthy Pregnancy, que tinha como objetivo reduzir as taxas de partos prematuros. Ao término da pesquisa, observou-se que essa ferramenta é um método efetivo para acompanhar as mulheres grávidas de alto risco, pois o acompanhamento das gestantes de forma regular, por meio do aplicativo e consultas presenciais, ajudou na identificaçáo precoce dos fatores de risco para o parto prematuro e, consequentemente, diminuiu as taxas de partos antes das 37 semanas de gestação. ${ }^{(6)}$

Aplicativos móveis também foram desenvolvidos para subsidiarem as mulheres no ciclo puerperal, principalmente no suporte à amamentação. Ao avaliar a usabilidade e utilidade de um aplicativo de telefonia móvel intitulado Moom Mãe, desenvolvido na Tailândia por meio de uma pesquisa quantitativa e qualitativa, com uma amostra de 21 mulheres, constatou-se um aumento no índice de nutrizes em aleitamento materno exclusivo e retardando o desmame precoce. ${ }^{(26)}$ Tal resultado vai ao encontro 
de um estudo prospectivo e observacional desenvolvido com oito mulheres na cidade de Quezon, nas Filipinas, com aplicativo móvel destinado a promover e melhorar a prática da amamentação, onde se constataram repercussóes positivas para as mulheres e seus companheiros, pois, além dos registros sobre o aleitamento materno, o dispositivo apresentava endereços de lugares públicos confortáveis para a prática, esclarecia dúvidas sobre a doação de leite e disponibilizava link de comunicação entre os bancos de leite e o posto de coleta de leite humano. ${ }^{(21)}$

Os aplicativos educacionais são válidos no apoio às mulheres no período pós-parto, visto que auxiliam na identificação de mudança não fisiológica e nos cuidados durante o período puerperal. ${ }^{(21)} \mathrm{Com}$ isso, acredita-se que o aplicativo Gestação Saudável é de bastante relevância, o qual também abrange o ciclo puerperal, possibilitando auxiliar essas mulheres para uma melhor adaptação na fase do puerpério, incluindo orientaçóes em relação aos cuidados com o recém-nascido, contribuindo na melhoria dos indicadores de saúde materno-infantil.

Destaca-se a abrangência e integralidade de conteúdos no aplicativo "Gestação Saudável", englobando o ciclo gravídico, trabalho de parto, puerpério e cuidados com o recém-nascido, evitando um cuidado compartimentalizado. O menu fale conosco emerge como outro grande diferencial, possibilitando uma aproximaçáo entre paciente/profissional e facilitando o acesso à serviços de saúde.

Para elaboração desse aplicativo, buscou-se, inicialmente, identificar, por meio de uma revisão de literatura, as principais informaçóes que poderiam compor um aplicativo móvel para promover o cuidado de mulheres na gestação e, em seguida, sua validação de conteúdo por especialistas. Ressalta-se que o desenvolvimento de aplicativos móveis, de maneira coerente e adequada, pressupóe a identificação das reais necessidades dos usuários e, posteriormente, sua validação. ${ }^{(20-22)}$

No que concerne à validação de conteúdo, pode-se observar, por meio da avaliaçáo dos especialistas - enfermeiros obstetras -, que o aplicativo oferece linguagem clara, compreensível e adequada, conteúdo relevante e importância prática. A validação de conteúdo do aplicativo Gestação Saudável mostrou a sua confiabilidade através IVC após a segunda rodada Delphi, sendo esse índice com resultado adequado para todos os itens avaliados pelos juízes. $\mathrm{O}$ IRA ideal demonstrou que houve homogeneidade nas avaliaçóes realizadas pelos especialistas. Com o teste de Mann-Whitney, observaram-se evidências de diferença estatística do Delphi 1 com o 2 para cada tela, identificando-se melhor avaliação na Delphi 2.

Os IVCs adequados demonstraram que o conteúdo abordado no aplicativo e a parte técnica do sistema são confiáveis, demonstram-se válidos. Com a confiabilidade apresentada, torna-se possível indicar o seu uso para gestantes como forma de contribuir para o seu conhecimento e melhoria do cuidado pré-natal realizado, uma vez que pode facilitar a comunicação entre profissionais e usuárias, aumentar a adesão nas consultas de pré-natal, oportunizando o diagnóstico precoce de intercorrências que possam surgir e incidir na diminuição de morbimortalidade materna e perinatal por causas preveníveis.

Os resultados de validação do instrumento, neste estudo, assemelham-se aos de uma pesquisa realizada na região Nordeste do Brasil sobre validação de cartilha para gestantes submetidas à raquianestesia na cirurgia cesariana, na qual avaliaram-se itens relacionados a linguagem e conteúdo, os quais apresentaram IVCs adequados. Para existir efeitos positivos quanto à usabilidade de uma ferramenta, a linguagem utilizada precisa ser clara e compreensível pelo público-alvo, além de apresentar relevância prática. ${ }^{(27)}$

Para os especialistas da tecnologia da informação e comunicação, no que diz respeito à organização e interface das telas e conteúdo do sistema, o dispositivo apresenta recursos claros, de rápido acesso e fáceis de manuseio para os usuários da tecnologia. Dessa forma, destaca-se a importância da viabilidade da tecnologia. Esses especialistas avaliaram positivamente os itens funcionalidade, confiabilidade, eficiência e usabilidade do aplicativo para gestantes, demonstrando que as informações fornecidas no aplicativo foram consideradas pertinentes, podendo ser utilizado por grávidas como ferramenta de promoção à saúde no período gravídico-puerperal, o que foi constatado por meio do valor do IVC apresentado. No Delphi 2, todos os itens avaliados por 
esses profissionais apresentaram IVCs adequados para validade de conteúdo e IRA ideal. Esse dado corrobora com pesquisa metodológica realizada no Brasil, a qual construiu e validou uma tecnologia educacional para gestantes de acordo com os mesmos critérios. Os resultados da avaliação da ferramenta, por 47 especialistas, demonstraram IVCs adequados. ${ }^{(28)}$ Assim, o aplicativo Gestação Saudável apresenta-se também com excelência legitimidade e credibilidade em seu recurso tecnológico.

A validação de tecnologias educativas por profissionais com experiência em avaliação de conteúdos em aplicativos é importante, visto que eles possuem um olhar mais aguçado para aspectos que podem influenciar o processo de aprendizagem, empoderamento e autocuidado. ${ }^{(29-31)}$ Ressalta-se que o resultado deste estudo obteve o consenso dos especialistas.

$\mathrm{Na}$ elaboração de aplicativos móveis, torna-se necessário incluir informaçóes suficientes, conceitos claros e coerência em relação ao tema a ser trabalhado para garantia de sua eficiência. ${ }^{(28)}$ Neste estudo, houve preocupaçáo em disponibilizar perguntas e respostas de forma clara, com uso de vocabulário simples e informaçóes suficientes, por meio de textos curtos para evitar a ambiguidade e permitir a transmissão e captação das mensagens. Esse dado corrobora com achados de pesquisa acerca da validaçáo de manual educativo para acompanhantes durante a gravidez, que obteve avaliação satisfatória quanto à clareza, objetividade e atratividade da linguagem. ${ }^{(29,30)}$

Apesar da validação de conteúdo do aplicativo indicar a confiabilidade das informaçóes e a importância dos assuntos abordados para o conhecimento das mulheres que se encontram no período gestacional, a pesquisa apresentou-se como limitação a não validação da ferramenta quanto a funcionalidade e usabilidade pelas gestantes, o que poderia implicar na restrição do seu uso por dificuldades no manuseio dos menus e falta de compreensão em relação às informaçóes disponibilizadas.

\section{Conclusão}

O IVC geral adequado entre os especialistas evidencia o aplicativo "Gestação saudável” como uma fer- ramenta que apresenta informaçóes confiáveis a ser utilizada no cuidado durante o período gravídico-puerperal, podendo potencializar o conhecimento das gestantes sobre a gravidez e puerpério, incentivar o autocuidado e orientaçáo em alguns cuidados com o recém-nascido, fortalecer a adesão às consultas de pré-natal e a acessibilidade ao contato com os profissionais. Ressalta-se que o aplicativo desenvolvido possibilita aquisição de conhecimento sobre direitos reprodutivos e participação na elaboração do plano de parto; o armazenamento de dados pessoais, das consultas e dos exames laboratoriais e imagens; lembrar as datas das consultas de pré-natal e esclarecer dúvidas pelo menu fale conosco. Ademais, essa tecnologia poderá contribuir para a prática do aleitamento materno, cuidados com recém-nascido, reforçando informaçóes sobre consultas de crescimento e desenvolvimento, higiene, limpeza do coto umbilical, testes de triagem neonatal e vacinação. Conteúdos, esses, validados com IVC adequado, pelos especialistas obstetras. A tecnologia desenvolvida pode ser usada, também, por profissionais de saúde como ferramenta educacional, uma vez que os conteúdos oferecidos no aplicativo são estratégias importantes para as mulheres conhecer as transformaçóes que ocorrem no corpo, os sinais de alerta e a relevância do aleitamento materno e cuidados com o recém-nascido. As informaçóes fornecidas através do aplicativo poderão ser um meio para o fortalecimento dos direitos reprodutivos.

\section{Colaborações}

Souza FMLC, Santos WN, Dantas JC, Sousa HRA, Moreira OAA e Silva RAR contribuíram com a concepção do estudo, análise e interpretação dos dados, redação do artigo, revisão crítica relevante do conteúdo intelectual e aprovação da versão final a ser publicada.

\section{Referências}

1. Barra DC, Almeida SR, Sasso GT, Paese F, Rios GC. Metodologia para modelagem e estruturação do processo de enfermagem informatizado em terapia intensiva. Texto Contexto Enferm. 2016;25(3):e2380015. 
2. Roberts S, Chaboyer W, Gonzalez R, Marshall A. Using technology to engage hospitalised patients in their care: a realist review. BMC Health Serv Res. 2017;17(1):388.

3. Banos O, Villalonga C, Garcia R, Saez A, Damas M, Holgado-Terriza JA, et al. Design, implementation and validation of a novel open framework for agile development of mobile health applications. Biomed Eng Online. 2015;14 Suppl 2:S6.

4. Mellado CM, Ávila IY. Need factors associated with the adequate use of prenatal control. Rev Cuid (Bucaramanga). 2016;7(2):1345-51.

5. Saavedra JS, Cesar JA, Linhares A0. Prenatal care in Southern Brazil: coverage, trend and disparities. Rev Saude Publica. 2019;53(40):40.

6. Krishnamurti T, Davis AL, Wong-Parodi G, Fischhoff B, Sadovsky Y, Simhan HN. Development and Testing of the MyHealthyPregnancy App: A Behavioral Decision Research-Based Tool for Assessing and Communicating Pregnancy Risk. JMIR Mhealth Uhealth. 2017;5(4):e42.

7. Kennelly MA, Ainscough K, Lindsay KL, O'Sullivan E, Gibney ER, McCarthy $\mathrm{M}$, et al. Pregnancy exercise and nutrition with smartphone application support: a randomized controlled trial. Obstet Gynecol. 2018;131(5):818-26.

8. Ledford CJ, Canzona MR, Cafferty LA, Hodge JA. Mobile application as a prenatal education and engagement tool: A randomized controlled pilot. Patient Educ Couns. 2016;99(4):578-82.

9. Dalton JA, Rodger D, Wilmore M, Humphreys S, Skuse A, Roberts CT, et al. The Health-e Babies App for antenatal education: feasibility for socially disadvantaged women. PLoS One. 2018;13(5):e0194337.

10. Hackett K, Lafleur C, Nyella P, Ginsburg O, Lou W, Sellen D. Impact of smartphone-assisted prenatal home visits on women's use of facility delivery: results from a cluster-randomized trial in rural Tanzania. PLOS One. 2018;13(6):e0199400.

11. Lee $Y$, Moon M. Utilization and content evaluation of mobile applications for pregnancy, birth, and child care. Healthc Inform Res. 2016;22(2):73-80.

12. McCulloh RJ, Fouquet SD, Herigon J, Biondi EA, Kennedy B, Kerns E, et al. Development and Implementation of a Mobile Device-Based Pediatric Electronic Decision Support Tool as Parto $\mathrm{f}$ a National Practice Standardization Project. J Am Med Inform Assoc. 2018; 25(9):117582.

13. Barra DC, Paim SM, Dal Sasso GT, Colla GW. Methods for developing mobile apps in health: an integrative review of the literature. Texto Contexto Enferm. 2017;26(4):e2260017.

14. McMillan SS, King M, Tully MP. How to use the nominal group and Delphi techniques. Int J Clin Pharm. 2016;38(3):655-62.

15. Pasquali L. Instrumentação psicológica: Fundamentos e práticas. Porto Alegre, Brasil: Artmed; 2010.

16. Pereira RD, Alvim NA. Delphi technique in dialogue with nurses on acupuncture as a proposed nursing intervention. Esc Anna Nery. 2015;19(1):174-80.
17. Vêscovi SJ, Primo CC, Sant' Anna HC, Bringuete ME, Rohr RV, Prado TN, et al. Mobile application for evaluation of feet in people with diabetes mellitus. Acta Paul Enferm. 2017;30(6):607-13.

18. Giannini SP, Latorre MR, Ferreira LP. Condition of Vocal ProductionTeacher questionnaire: comparison of responses on Likert scale and visual analog scale. CoDAS. 2016;28(1):53-8.

19. Rubio DM, Berg-Weger M, Tebb SS, Lee ES, Rauch S. Objectifying content validity: conducting a content validity study in social work research. Soc Work Res. 2003;27(2):94-111.

20. Almeida RF, Sousa TJ, Couto AS, Marques AJ, Queirós CM, Martins CL. Development of we Cope, a mobile app for illness self-management in schizophrenia. Arch Clin Psychiatry. 2019;46(1):1-4.

21. Marko KI, Krapf JM, Meltzer AC, Oh J, Ganju N, MartinezAG, et al. Testing the Feasibility of Remote Patient Monitoring in Prenatal Care Using a Mobile App and Connected Devices: A Prospective Observational Trial. JMIR Res Protoc. 2016;5(4):e200.

22. Silva RM, Brasil CC, Bezerra IC, Queiroz FF. Uso de tecnologia móvel para o cuidado gestacional: avaliação do aplicativo GestAção. Rev Bras Enferm. 2019; 72 (Suppl 3):266-73.

23. Kobayashi S, Hanada N, Matsuzaki M, Takehara K, Ota E, Sasaki $H$, et al. Assessment and support during early labour for improving birth outcomes. Cochrane Database Syst Rev. 2017;4(2):CD011516.

24. Smith CA, Levett KM, Collins CT, Dahlen HG, Ee CC, Suganuma M. Massage, reflexology and other manual methods for pain management in labour. Cochrane Database Syst Rev. 2018 Mar 28;3(3):CD009290.

25. Dodou HD, Oliveira TD, Oriá MO, Rodrigues DP, Pinheiro PN, Luna IT. Educational practices of nursing in the puerperium: social representations of puerperal mothers. Rev Bras Enferm. 2017;70(6):1250-8.

26. Balogun 00, O'Sullivan EJ, McFadden A, Ota E, Gavine A, Garner CD, et al. Interventions for promoting the initiation of breastfeeding. Cochrane Database Syst Rev. 2016 Nov;11(2):CD001688.

27. Pinto SL, Lisboa KW, Neto NM, Sampaio LA, Oliveira MF, Caetano JÁ. Patient positioning for spinal anesthesia: construction and validation of a flipchart. Acta Paul Enferm. 2018;31(1):25-31.

28. Rodrigues TC, Teles LF. 0 uso de mensagens eletrônicas instantâneas como recurso didático. Rev Bras Estud Pedagog. 2019;100(254):17-38.

29. Goetz M, Müller M, Matthies LM, Hansen J, Doster A, Szabo A, et al. Perceptions of patient engagement applications during pregnancy: a qualitative assessment of the patient's perspective. JMIR Mhealth Uhealth. 2017;5(5):e73.

30. Stewart JJ, Fayed I, Henault S, Kalantar B, Voyadzis JM. Use of a Smartphone Application for Spine Surgery Improves Patient Adherence with Preoperative Instructions and Decreases Last-minute Surgery Cancellations. Cureus. 2019;11(3):e4192.

31. Melo EB, Primo CC, Romero WG, Sant'Anna HC, Sequeira CA, Lima EF, et al. Construção e validação de aplicativo móvel para o desenvolvimento de histórico e diagnóstico de enfermagem. Rev Bras Enferm. 2020; 73(6):e20190674. 